\title{
Análisis de los factores del rendimiento académico de los estudiantes de Pedagogía desde la técnica de grupo nominal y método Delphi
}

\section{Analysis of university academic achievement conducted among general education students and based on the nominal group technique and the Delphi method}

\author{
Margarita R. Rodríguez-Gallego ${ }^{1}$ \\ margaguez@us.es \\ Universidad de Sevilla, España
}

\section{Resumen:}

Desde hace varios cursos académicos, profesores del Departamento de Didáctica y Organización Educativa que imparten Didáctica General, en la Facultad de Ciencias de la Educación de la Universidad de Sevilla, han detectado un alto índice de suspensos y no presentados en esta asignatura. Fruto de esta preocupación surgió la necesidad de conocer y analizar las causas de este bajo rendimiento.

Este estudio es exploratorio y el muestreo utilizado ha sido no probabilístico, por cuotas, con un número de individuos que reunían determinadas condiciones. El total de estudiantes que han participado en la Técnica de Grupo Nominal han sido de 20, seleccionados entre los 312 estudiantes matriculados en el primer curso, de la titulación de Pedagogía. Para el método Delphi han colaborado diez profesores

\begin{abstract}
:
For the last academic years, professors at the Department of Education and Teaching Organization in charge of the General Education subject in the Faculty of Educational Sciences of the University of Seville, have realized that there are high failure and not-attendance-to-exam rates in this subject. In light of the seriousness of the situation, it is necessary to understand and analyze the causes of this poor achievement. The study is exploratory and the method applied is the nonprobability quota sampling. The sample in the Nominal Group Technique is 20 students selected amongst a total of 312 registered in the first year of the General Education degree. 10 expert professors in the subject have collaborated for the Delphi method. Data show that students attribute their
\end{abstract}

\footnotetext{
1 Dirección para correspondencia (Correspondence address):

Margarita R. Rodríguez-Gallego. Dpto. de Didáctica y Organización Educativa. Facultad de Ciencias de la Educación. Universidad de Sevilla. C/ Pirotecnia, s/n. 41013 Sevilla (España).
} 
Análisis de los factores del rendimiento académico de los estudiantes de Pedagogía desde la técnica de grupo nominal y método Delphi

Margarita R. Rodríguez-Gallego

expertos que imparten esta asignatura en esta titulación.

Los datos revelan que los estudiantes achacan su bajo rendimiento, en un primer momento a los profesores, seguido de su falta de actitudes para el estudio. Para los profesores la variable que más incide en el bajo rendimiento es la dificultad de la materia, técnicas de estudio inadecuadas, desmotivación y excesivo número de asignaturas. Entre las propuestas de mejora cabe destacar la implicación de la institución con una revisión de los planes de estudio, adquirir estrategias y técnicas de motivación por parte de los profesores y los alumnos mejorar sus hábitos, técnicas de estudio y asistir a clase.

\section{Palabras clave:}

Educación Superior; rendimiento académico; técnica de grupo nominal; método Delphi. poor achievement, in the first place, to the teachers of the subject, and then to his poor willingness to study. For teachers, the variables that most influence students poor achievement are: the difficulty of the subject, inadequate study skills, motivation, and excessive number of subjects. As conclusions, we have to outline the need to improve the study programmes and the strategic and motivational techniques on the part of teachers. Students, in turn, should improve their study habits, study techniques, and attend classes more frequently.

\section{Key words:}

Higher Education; academic achievement; nominal group technique; Delphi method.

\section{Résumé:}

Pour un certain nombre de cours universitaires, professeurs au Département de Curriculum et Organisation Scolaire qui enseignent Didactique Générale, de la Faculté des Sciences de l'éducation de l'Université de Séville, viennent souligner un taux élevé d'échecs. Le résultat de cette préoccupation est né de la nécessité de comprendre et d'analyser les causes de ce mauvais rendement.

C'est une étude exploratoire, avec un certain nombre de personnes qui répondaient à des conditions. Le nombre total d'élèves qui ont participé à la Technique du Groupe Nominal 20 ont été sélectionnés parmi les 312 étudiants inscrits au premier cours de Pédagogie. Pour la méthode Delphi ont travaillé dix professeurs qui enseignent la matière. Les données révèlent que les élèves attribuent leur faible rendement, dans un premier temps les professeurs qui enseignent la matière, suivi par son manque d'attitudes à l'étude. Pour les professeurs la variable qui exerce une plus grande influence sur le mauvais rendement est la difficulté de la matière, le manque des techniques d'étude, la motivation et le nombre excessif des matières.

\section{Mots clés:}

L'Enseignement Supérieur; le rendement universitaire; la technique du groupe nominal; méthode Delphi.

Fecha de recepción: 24-8-2013

Fecha de aceptación: 27-2-2014 


\section{Fundamentación teórica}

Este estudio ha sido diseñado para identificar, analizar y establecer propuestas de mejora sobre algunos factores determinantes del rendimiento académico, en la Facultad de Ciencias de la Educación de la Universidad de Sevilla, en la asignatura Didáctica General del primer curso del Grado de Pedagogía.

De los 312 estudiantes matriculados en esta asignatura, en el curso académico 2011-2012, se alcanzó un índice de no presentados del $60.9 \%$ y de suspensos del $17.2 \%$.

El alto índice de fracaso escolar en esta asignatura, la deserción y abandono, las bajas voluntarias y el bajo rendimiento académico es un problema que comparte nuestra Universidad y la mayoría de Universidades españolas.

Para abordar este problema ha sido necesario plantear, en primer lugar, que se entiende por rendimiento académico, determinar las variables que lo provocan y así poder establecer un plan de apoyo para los estudiantes.

El rendimiento académico es un constructo muy complejo, multivariado y multifactorial causado por elementos cognitivos, actitudinales, socio-ambientales, institucionales y pedagógico/metodológicos. Tejedor y García-Valcárcel (2007) plantean que lo habitual es identificar rendimiento con resultados, determinados por las calificaciones que obtienen los alumnos durante su carrera hasta la obtención del título correspondiente. Este estudio se ha centrado, siguiendo a Tejedor y García-Valcárcel (2007: 445), en el rendimiento en sentido estricto, medido a través de la presentación a exámenes o éxito en las pruebas (calificaciones), que se traduce en unas determinadas tasas de promoción, repetición y abandono. También se considera el término de regularidad académica, las tasas de presentación o no a las convocatorias de exámenes y las actitudes de los estudiantes.

Aunque se debe considerar que la percepción de los estudiantes sobre su rendimiento académico no está necesariamente asociado con la evaluación formal que obtienen a partir de las actividades que ellos realizan, sino más bien integra numerosas variables que representan diferentes categorías de análisis que puede incluir el autoconcepto académico como determinante fundamental del proceso. Con esto se hace hincapié, con Montero, Villalobos y Valverde (2007: 217) que, el rendimiento académico no es el producto analítico de una única aptitud, sino más bien el 
Análisis de los factores del rendimiento académico de los estudiantes de Pedagogía desde la técnica de grupo nominal y método Delphi

Margarita R. RodríGuez-Gallego

resultado sintético de una suma (nunca bien conocida) de elementos que actúan en, y desde la persona que aprende, tales como factores cognitivos, actitudinales, socio-ambientales, institucionales y pedagógico/ metodológicos.

En la mayoría de los países de nuestro entorno económico-cultural, como se ha comentado, existe el problema del bajo rendimiento en sus Universidades, ya se midan éstos a través de las tasas de abandono-éxito, regularidad académica o calificaciones obtenidas. Además la mayor parte de los abandonos, para Tejedor y García-Valcárcel (2007), se produce en los primeros años de carrera, fundamentalmente en primero (en torno al $65 \%$ de abandono) y algo en segundo (en torno al $20 \%$ ).

Las investigaciones llevadas a cabo en Universidades nacionales e internacionales ponen de manifiesto los numerosos factores que afectan al rendimiento académico. Entre ellos, una buena combinación entre las preferencias de aprendizaje de los alumnos y el estilo de enseñanza del profesor han demostrado tener efectos positivos en el rendimiento de los estudiantes (Harb y El-Shaarawi, 2006; Pashler et. al, 2008; Aripin et. al, 2008; Adunola, 2011; Bharadwaj y Pal, 2011; Kang'ahi et al., 2012; Ganyaupfu, 2013). El aprendizaje de preferencias se refiere a una manera natural y habitual de la persona para asimilar nueva información. En efecto, en la investigación realizada por Omrod (2008) Ilegaron a la conclusión que algunos estudiantes parecen aprender mejor cuando se presenta la información a través de palabras (aprendices verbales), mientras que otros parecen aprender mejor cuando se presenta en forma de imágenes (aprendices visuales) y otros a través del movimiento (aprendices kinestésicos). Es evidente que en una clase donde sólo se emplea un método de instrucción, hay una gran posibilidad que un elevado número de estudiantes no encuentren el ambiente de aprendizaje óptimo, lo que podría afectar a su rendimiento académico.

En la investigación realizada por Akiri y Ugborugbo (2009) la competencia docente es determinante en el rendimiento de los estudiantes. Es un constructo multidimensional que debe medir una variedad de aspectos relacionados entre sí, que incluye, ser experto en la materia, preparar las clases, presentar la información y ser un comunicador eficaz. Es lo que Yániz (2006) denomina planificar la enseñanza.

Por su parte, Romer (1993) es uno de los primeros autores en investigar la relación entre la asistencia y rendimiento académico. La investigación sobre este tema parece proporcionar un consenso en la correlación 
existente entre estudiantes que faltan a clases y rendimiento académico bajo en comparación con los que asisten a clases (Devadoss y Foltz, 1996; Durden y Ellis, 1995; Park y Kerr, 1990; Harb y El-Shaarawi, 2006).

En cuanto al sexo no hay acuerdo en los estudios realizados. En algunas investigaciones se recoge que las mujeres tienen un mayor rendimiento que los varones y en otras que no existen diferencias apreciables. En muchos casos, esta variabilidad está asociada a la carrera elegida.

Otros trabajos empíricos revisados demuestran que variables no cognitivas como, las expectativas, características personales, familia, estado civil, integración social y académica, hábitos de estudio, tamaño de la clase y ayuda financiera, entre otros, son factores importantes que influyen en el progreso del estudiante (Smith y Naylor, 1993; Keil y Partell, 1997; Díaz, 2003; Alonso, 2005; Lassibille, 2011; Yousuf y Ranjha, 2011; Al-Mutairi, 2011).

Karemera (2003) encontró que el rendimiento de los alumnos tiene una correlación significativa con respecto a la manera en que el plan de estudios de las titulaciones está diseñado (excesivo número de asignaturas, descriptores inadecuados, troncalidad, número de créditos y carácter anual o cuatrimestral).

Otro factor determinante del rendimiento son las pruebas específicas sobre la admisión de estudiantes. Éstas pruebas han sido históricamente utilizadas como predictor del éxito académico en la enseñanza superior, así como la aplicación efectiva de los criterios mínimos de admisión y la prestación de la debida orientación a los estudiantes universitarios (Durr, 1999; Rhodd et al., 2000; Silva et al., 2010).

En definitiva, conocer los diferentes factores que inciden en el rendimiento académico de los universitarios favorece un enfoque más completo para la toma decisiones y la mejora de la calidad educativa de las instituciones.

A modo de síntesis, en la Figura 1 quedan recogidas, las posibles variables que pueden ser determinantes en el estudio y análisis del rendimiento universitario.

\begin{tabular}{ll}
\hline TIPO DE FACTOR & VARIABLES \\
\hline Demográfica & Sexo, edad, estado civil, financiación estudios \\
\hline Sociocultural & $\begin{array}{l}\text { Origen sociocultural, nivel educativo padre y madre, cli- } \\
\text { ma educativo familiar, integración social del estudiante }\end{array}$ \\
\hline
\end{tabular}


Análisis de los factores del rendimiento académico de los estudiantes de Pedagogía desde la técnica de grupo nominal y método Delphi

Margarita R. RodríGuez-Gallego

\begin{tabular}{ll}
\hline TIPO DE FACTOR & VARIABLES \\
\hline Cognitiva & $\begin{array}{l}\text { Aptitudes intelectuales, capacidades y habilidades cog- } \\
\text { nitivas y metacognitivas, estilos cognitivos }\end{array}$ \\
\hline Actitudinal & $\begin{array}{l}\text { Responsabilidad hacia el aprendizaje, satisfacción, inte- } \\
\text { rés por los estudios, decisión ante los estudios, motiva- } \\
\text { ción, hábitos de estudio, habilidades sociales, autocon- } \\
\text { cepto académico }\end{array}$ \\
\hline Académica & $\begin{array}{l}\text { Tipo de estudios, curso, opción en la que estudia la ca- } \\
\text { rrera, rendimiento previo, número de créditos matricula- } \\
\text { dos, nota de acceso a la Universidad, asistencia a clase, } \\
\text { satisfacción/abandono con respecto a los estudios }\end{array}$ \\
\hline $\begin{array}{l}\text { Pedagógica/meto- } \\
\text { dológica }\end{array}$ & $\begin{array}{l}\text { Expectativas y actitudes del profesor, formación y expe- } \\
\text { riencia del profesor, personalidad del profesor, tamaño } \\
\text { del grupo, clima de clase, metodología de enseñanza, } \\
\text { estrategias de evaluación }\end{array}$ \\
\hline Institucional & $\begin{array}{l}\text { Tipo y tamaño del centro, funcionamiento institución, } \\
\text { condiciones institucionales, servicios institucionales de } \\
\text { apoyo, ambiente estudiantil, complejidad de los estu- } \\
\text { dios, políticas educativas }\end{array}$ \\
\hline Figura 1. Variables que influyen en el rendimiento académico en la Universidad.
\end{tabular}

En este estudio, para identificar y analizar los factores determinantes del rendimiento de los estudiantes, se han utilizado dos técnicas de educción de conocimientos: la Técnica de Grupo Nominal (TGN) para conocer las percepciones de los estudiantes y el método Delphi para conocer las opiniones de los profesores.

La Técnica de Grupo Nominal es un método especial, para administrar en una reunión orientada a la resolución de problemas o toma de decisiones, que se presta especialmente para la generación de ideas y su evaluación con el propósito de establecer prioridades. Para ello, trata de guiar el proceso de decisión asegurando la participación de todos los miembros, la ponderación equilibrada de las ideas e incorpora un procedimiento de agregación para ordenar las propuestas que puedan surgir. Los miembros del grupo están físicamente presentes pero operan de forma independiente. Cada miembro aporta sus decisiones para sumar después los resultados y utilizar la votación como medio de conseguir una valoración grupal. Esta técnica ha sido utilizada para el análisis de las relaciones laborales en empresas (Van der Waal y Uys, 2009), servicios sanitarios (Bernal-Delgado, Peiró y Sotoca, 2006), gestión de la calidad 
en Educación Superior (Abdullah e Islam, 2011), relaciones familiares (Sánchez, Landeta e Iturralde, 2012) y educación (Morales y Fraca, 2002; Rodríguez-Gallego, 2007).

En cuanto al método Delphi se basa en el principio de la inteligencia colectiva y trata de lograr un consenso de opiniones expresadas individualmente por un grupo de personas seleccionadas cuidadosamente como expertos cualificados en torno al tema, por medio de la iteración sucesiva de un cuestionario retroalimentado. La relevancia de esta técnica se puede apreciar en las áreas de aplicación tan variadas, distinguiéndose hasta 21 áreas diferentes, siendo las tres principales, servicios de salud (Yáñez y Cuadra, 2008; Balasubramanian y Agarwal, 2012; García y Suárez, 2013); educación (Landeta, 2006; Sandrey y Bulger, 2008; Van der Schaaf y Stokking, 2011; Aharony y Bronstein, 2013) y empresa (Sánchez, Landeta e Iturralde, 2012). Recientemente se viene utilizando el método Delphi (Cantón, Valle y Arias, 2008; Mira et al., 2012), y la Técnica de Grupo Nominal (Abdullah e Islam, 2011) para el gobierno y gestión de las Universidades.

\section{Diseño de la investigación y metodología}

\subsection{Objetivo}

El objetivo de nuestro estudio ha sido, por tanto, identificar y analizar los factores asociados al bajo rendimiento académico en la asignatura Didáctica General y realizar propuestas de mejora.

\subsection{Participantes}

Al ser un estudio exploratorio, el muestreo utilizado ha sido no probabilístico, por cuotas, con un número de individuos que reúnen unas determinadas condiciones. El total de estudiantes, del primer curso de esta asignatura en el curso académico 2011-2012, fue de 312. Para la Técnica de Grupo Nominal (TGN) se tomó la decisión de seleccionar a 20 estudiantes distribuidos en los cuatros grupos existentes (dos grupos de turno de mañana y dos de tarde). Con la siguiente distribución por cuotas: 
Análisis de los factores del rendimiento académico de los estudiantes de Pedagogía desde la técnica de grupo nominal y método Delphi

Margarita R. Rodríguez-Gallego

\begin{tabular}{|c|c|c|c|c|c|c|}
\hline SEXO & EDAD & TURNO & ESTADO CIVIL BECADO/A & $\begin{array}{r}\text { OP } \\
\text { ELEC } \\
\text { TITUI }\end{array}$ & $\begin{array}{l}\text { IÓN } \\
\text { CIÓN } \\
\text { ICIÓN }\end{array}$ & $\begin{array}{c}\text { NIVEL } \\
\text { SOCIO- } \\
\text { CULTURAL }\end{array}$ \\
\hline Mujeres 10 & $\begin{array}{c}\text { Menos } \\
\text { de } 20\end{array}$ & Mañana 10 & En pareja $10 \begin{array}{l}\text { Con } \\
\text { beca }\end{array} 10$ & 1 & 7 & $\begin{array}{c}\text { Medio } \\
\text { bajo }\end{array}$ \\
\hline \multirow[t]{2}{*}{ Hombres 10} & $20-3010$ & Tarde 10 & Soltero/a $10 \underset{\text { beca }}{\operatorname{Sin}} 10$ & 2 & 7 & $\begin{array}{l}\text { Medio } \\
\text { alto }\end{array}$ \\
\hline & $\begin{array}{c}\text { Más } \\
\text { de } 40\end{array}$ & & & 3 & 6 & \\
\hline TOTAL 20 & 20 & 20 & 20 & & 20 & 20 \\
\hline
\end{tabular}

Figura 2. Distribución de los estudiantes de la muestra por cuotas.

Los estudiantes menores de 20 años que se ajustaban a la distribución por cuotas y que habían sido seleccionados (5 sujetos) no quisieron participar en el estudio. Por este motivo, este grupo de edad está descompensado con respecto al resto.

En el método Delphi colaboraron siete profesoras y tres profesores que han impartido la asignatura, cinco con más de diez años de experiencia y cinco con menos de diez años de experiencia.

\subsection{Instrumentos}

En función del objetivo y del marco teórico en el que se sustenta el estudio, se utilizaron dos técnicas de educción de conocimientos para extraer la información necesaria de los dos colectivos implicados. La Técnica de Grupo Nominal para conocer las percepciones de los estudiantes a través de una pregunta abierta, consensuada por el equipo técnico (20 profesores del Departamento de Didáctica y Organización Educativa), con cuatro rondas y para el método Delphi un cuestionario validado por el equipo técnico, con 13 ítems, y contestado, en dos rondas, por los expertos. En la Figura 3 queda recogido el proceso de recogida de información. 


\begin{tabular}{|c|c|c|c|c|}
\hline INSTRUMENTOS & SELECCIÓN PREGUNTAS & CRITERIOS & RONDAS & MODALIDAD \\
\hline $\begin{array}{l}\text { Técnica } \\
\text { de Grupo } \\
\text { Nominal }\end{array}$ & $\begin{array}{l}\text { Equipo técnico } \\
1 \text { pregunta abierta }\end{array}$ & Categorías & $\begin{array}{c}\mathbf{1}^{\mathbf{a}} \text { ronda: } \\
\text { categorizar } \\
\mathbf{2}^{\mathbf{a}} \text { ronda: } \\
\text { categorizar } \\
\mathbf{3}^{\mathbf{a}} \text { ronda: } \\
\text { categorizar } \\
\text { y ordenar } \\
\mathbf{4}^{\mathbf{a}} \text { ronda: } \\
\text { ranking }\end{array}$ & $\begin{array}{l}\text { Presencial } \\
\text { Presencial } \\
\text { Presencial } \\
\text { Presencial }\end{array}$ \\
\hline $\begin{array}{l}\text { Método } \\
\text { Delphi }\end{array}$ & $\begin{array}{l}\text { Cuestionario elabo- } \\
\text { rado por el Equipo } \\
\text { técnico } \\
12 \text { preguntas cerradas } \\
(1-5) \text { y una abierta }\end{array}$ & $\begin{array}{l}\text { Unanimidad } \\
\text { Consenso } \\
\text { Discrepancia }\end{array}$ & $\begin{array}{l}\mathbf{1}^{\mathbf{a}} \text { ronda: } \\
\text { Pronósticos } \\
\mathbf{2}^{\mathbf{a}} \text { ronda: } \\
\text { Consenso }\end{array}$ & $\begin{array}{l}\text { e-mail } \\
\text { e-mail }\end{array}$ \\
\hline
\end{tabular}

Figura 3. Instrumentos de recogida de información.

\subsection{Procedimiento}

La ejecución de la investigación se desarrolló en cinco etapas o fases siguiendo a Morales y Fraca (2002):

Etapa I. Conformación de los participantes del Grupo Nominal (veinte estudiantes elegidos por cuotas). De forma paralela, en esta misma etapa, realizamos la selección del grupo de expertos para el Delphi (diez profesores doctores que imparten esta asignatura en el Grado de Pedagogía).

Etapa II. Planificación y conducción de la TGN. Esta etapa implicó la organización de la sesión de trabajo con el grupo nominal. En esta sesión se obtuvieron las listas preliminares de los planteamientos del grupo. La consigna inicial respondía al objetivo perseguido en el trabajo: ¿Cuáles son los principales problemas en esta asignatura para tan bajo rendimiento académico?. Esta pregunta fue consensuada por el equipo técnico que participaba en este estudio por considerarla directa y clara para los estudiantes.

Etapa III. Las ideas obtenidas en el grupo nominal, en la primera ronda, se sometieron a una exhaustiva revisión en cuanto a estilo y ortografía empleada. En la segunda ronda cada estudiante ha tenido la oportunidad de reconsiderar sus respuestas a la vista de su contestación inicial, añadir nuevas ideas y crear categorías. En esta segunda ronda no se añadieron nuevas ideas y se agruparon las ideas en dos categorías: pedagó- 
Análisis de los factores del rendimiento académico de los estudiantes de Pedagogía desde la técnica de grupo nominal y método Delphi

Margarita R. RodríGuez-Gallego

gica/metodológica e institucional. En la tercera ronda, no se añadieron ideas y se creó la categoría actitudinal y en la cuarta ronda la categoría académica. Se procedió a ordenar jerárquicamente las aportaciones más importantes, estableciendo un ranking de prioridades. Posteriormente al voto se abrió la discusión en la que se analizaron los resultados de la votación. En la Figura 4 quedan recogidas las fases de la TGN.

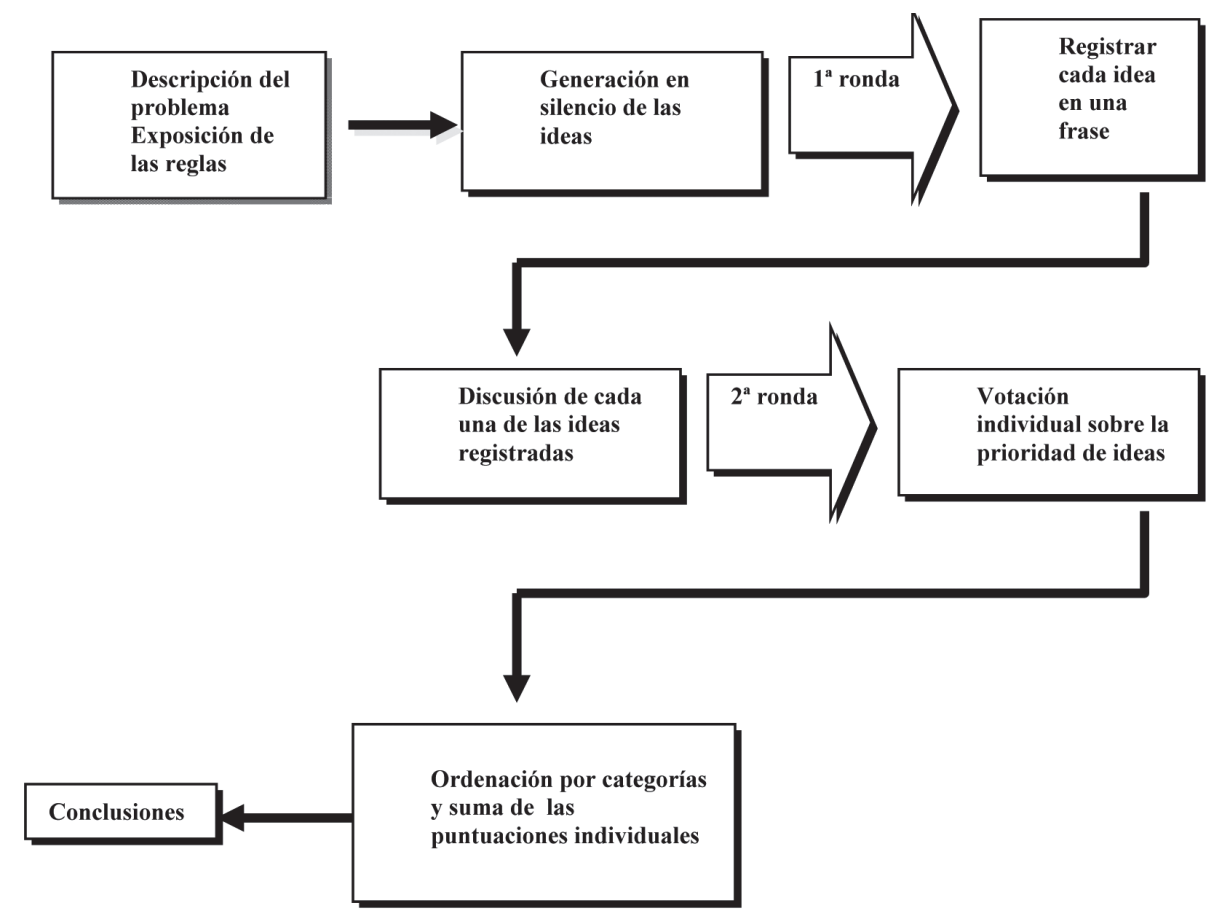

(3 y 4 rondas)

Figura 4. Proceso seguido en la Técnica de Grupo Nominal.

Etapa IV. Elaboración y aplicación de las rondas uno y dos del método Delphi. El cuestionario, validado por el equipo técnico, fue enviado, en $1^{\text {a }}$ ronda, por correo electrónico a los diez expertos, de los cuales todos aceptaron la invitación y lo devolvieron con sus pronósticos. El criterio elegido para decidir la valoración de las preguntas de la $1^{\text {a }}$ ronda, a efectos de interpretación de los resultados, quedó del siguiente modo:

a) Unanimidad: cuando todos los expertos efectúan idéntica respuesta.

b) Consenso: Cuando la mayoría de los expertos contestan la misma respuesta sin alcanzar unanimidad. 
c) Discordancia o discrepancia para el resto de situaciones posibles, es decir, cuando una misma respuesta o no es compartida por, al menos, las 2/3 partes de los expertos.

Antes de iniciar la $2^{\mathrm{a}}$ ronda cada experto ha recibido, por correo electrónico, el cuestionario con su respuesta anterior acompañado de las valoraciones anónimas de todos los participantes para poder contrastar sus criterios con los del resto del grupo y poder ofrecer nuevamente su juicio revisado. Con las respuestas individuales en relación con la posición global del colectivo, se espera que reconsideren sus predicciones con el propósito de ampliar el grado de acuerdo acerca de cada pregunta, y en el caso que no se produzca, consolidar las posiciones en las que se observen discrepancias manifiestas. En esta ronda, cada miembro ha tenido la oportunidad de reconsiderar sus respuestas a la vista de su contestación inicial y la posición global del conjunto de expertos, salvo en las cuestiones con respuestas unánimes y consensuadas en la primera ronda, cuya reconsideración se ha obviado.

En la $1^{a}$ ronda ha habido unanimidad en todas las preguntas, a excepción de dos de ellas, pregunta 10 (Se considera satisfecha/o con los resultados finales de la evaluación) y 12 (Exprese su valoración global del curso) que ha bajado de un 4 a un 3 . En la $2^{\text {a }}$ ronda no se ha llegado a un acuerdo en estas dos preguntas.

En la Figura 5 queda recogido el proceso seguido en el estudio a través del Delphi. 
Análisis de los factores del rendimiento académico de los estudiantes de Pedagogía desde la técnica de grupo nominal y método Delphi

Margarita R. RodríGuez-Gallego

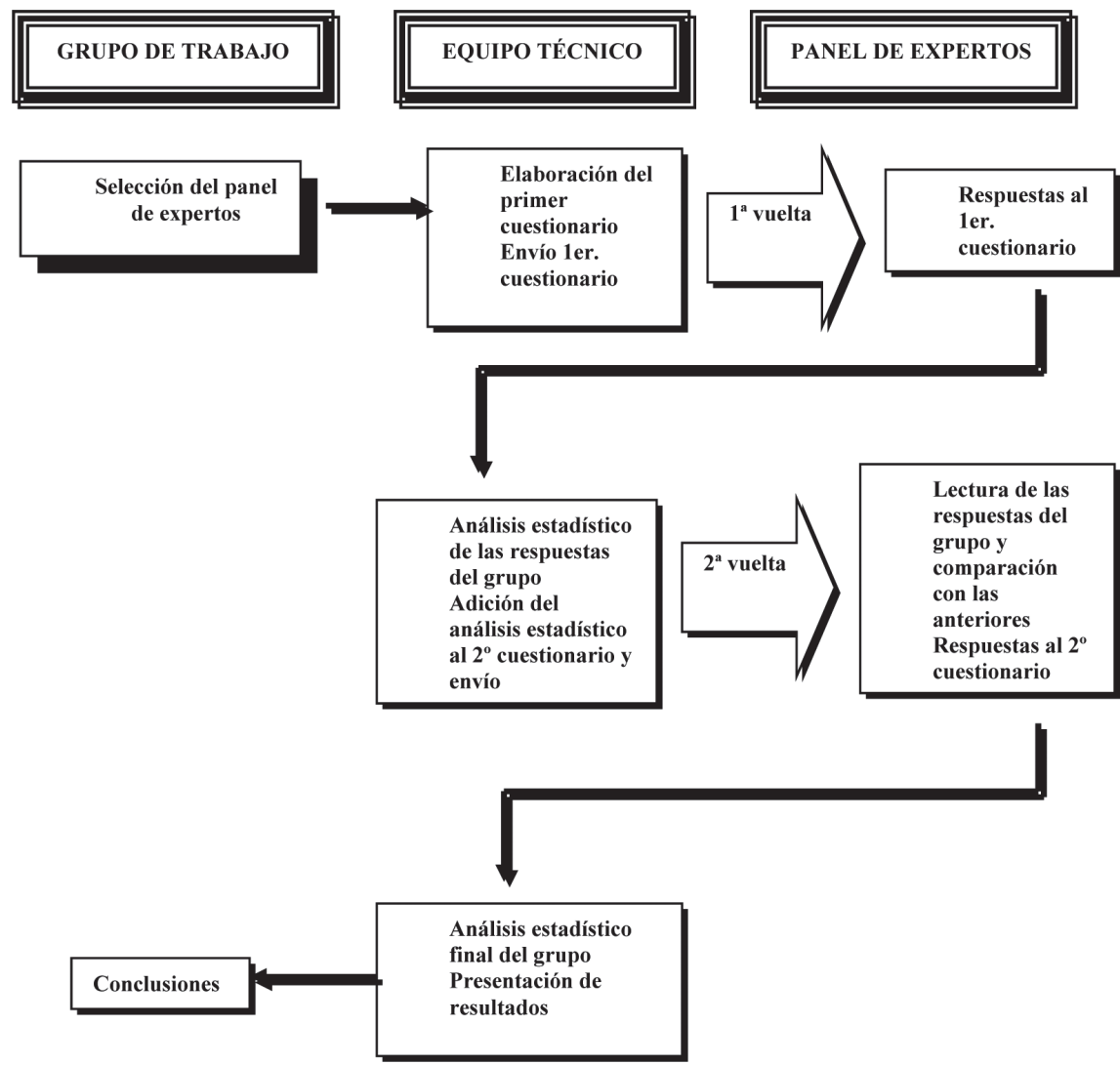

Figura 5. Proceso Delphi.

Etapa $V$. Posteriormente, se recogieron y procesaron estadísticamente los resultados para la estimación de los valores que permitieron establecer la tendencia de opinión y el consenso logrado en la consulta realizada a profesores y estudiantes.

\section{Resultados}

Los principales estadísticos empleados en el método Delphi han sido frecuencias, porcentajes, media y desviación típica (Keeney, Hasson y McKenna, 2001). Ello ha permitido dar una visión de conjunto de los resultados obtenidos en cada una de las preguntas. En la TGN frecuencias absolutas y relativas, porcentajes y ranking. 
Se consideran, en cierto modo, neutralizadas las variables demográfica y sociocultural, al haber seleccionado la muestra de los estudiantes y profesores por cuotas.

Las aportaciones realizadas por los estudiantes que han participado en la TGN, quedan recogidas en la Tabla 1.

Tabla 1. Ideas expresadas por los estudiantes en la TGN

\begin{tabular}{|c|c|c|c|}
\hline$N^{0}$ & Idea & Rango (1-5) & TOTAL \\
\hline 1 & Asignatura cuatrimestral & $3,3,4,2,5,2,3$ & 22 \\
\hline 2 & Falta coordinación entre asignaturas & $1,5,5,4,4,3,2,5,1$ & 30 \\
\hline 3 & Masificación en las aulas & 1 & \\
\hline 4 & Excesivo número de asignaturas & $5,5,5,4,4,3,4,3,5,3,2,4$ & 47 \\
\hline 5 & $\begin{array}{l}\text { Asignaturas impartidas por más de dos } \\
\text { profesores }\end{array}$ & $2,4,5,3,2,4,5,5,3,2$ & 35 \\
\hline 6 & No cursar la carrera en $1^{a}$ opción & $4,5,5,5,4,4,5,5$ & 37 \\
\hline 7 & Dificultad de la materia & $5,5,4,5,5,4,4,3,4$ & 39 \\
\hline 8 & $\begin{array}{l}\text { Subjetividad profesores en la evalua- } \\
\text { ción }\end{array}$ & $1,3,2,1$ & 7 \\
\hline 9 & Falta de orientación en los estudios & $2,3,1,4,3$ & 13 \\
\hline 10 & Clima competitivo entre compañeros & $1,3,1$ & 5 \\
\hline 11 & Excesiva exigencia del profesorado & $3,5,4,5,5,2,4,5,3,2$ & 38 \\
\hline 12 & Escasas perspectivas laborales & $3,4,5,5,2,5,5$ & 29 \\
\hline 13 & Desproporcionado número de trabajos & $2,5,5,5,4,5,5,5,5,5,3,1,5$ & 55 \\
\hline 14 & Falta de esfuerzo & $3,4,2,5,4,5$ & 23 \\
\hline 15 & Asignatura muy teórica & $5,5,5,4,5,4,3,5,5,4,2,1$ & 48 \\
\hline 16 & Créditos prácticos complicados & $4,3,2,4,1,2$ & 16 \\
\hline 17 & Poca motivación de los profesores & $1,5,4,3,3,4,2,1$ & 23 \\
\hline 18 & Poco tiempo para asimilar contenidos & $2,1,2,3$ & 8 \\
\hline 19 & Falta de claridad expositiva & $4,3,4,2,5$ & 18 \\
\hline 20 & Créditos prácticos poco adecuados & $1,2,4,2,1$ & 10 \\
\hline 21 & Falta de autocontrol y responsabilidad & $5,4,5,4,3,5,5,4,1,3,5$ & 44 \\
\hline 22 & Bajo nivel de conocimientos previos & $1,3,2,1$ & 7 \\
\hline 23 & Falta de asistencia a clase & $1,5,5,4,3,5,5,4,2,1,5$ & 40 \\
\hline 24 & Escasa comunicación con los alumnos & 1,2 & 3 \\
\hline 25 & Técnicas de estudio inadecuadas & $3,2,4,5,5,4,5,3,4,5$ & 40 \\
\hline
\end{tabular}


Análisis de los factores del rendimiento académico de los estudiantes de Pedagogía desde la técnica de grupo nominal y método Delphi

Margarita R. Rodríguez-Gallego

Una vez recogidas las aportaciones dadas por los estudiantes y frecuencias correspondientes, en la Tabla 2 se muestra el ranking de ideas.

Tabla 2. Factores que afectan al rendimiento académico desde la perspectiva de los estudiantes

\begin{tabular}{lcccc}
\hline \multicolumn{1}{c}{ Factor } & $\begin{array}{c}\text { Frecuencia } \\
\text { absoluta }\end{array}$ & $\begin{array}{c}\text { Frecuencia } \\
\text { relativa }\end{array}$ & \% & Orden \\
\hline $\begin{array}{l}\text { Desproporcionado número de } \\
\text { trabajos }\end{array}$ & 55 & 0.114 & 11.41 & 1 \\
\hline Asignatura muy teórica & 48 & 0.995 & 9.95 & 2 \\
\hline Excesivo número de asignaturas & 47 & 0.975 & 9.75 & 3 \\
\hline $\begin{array}{l}\text { Falta de autocontrol y responsabi- } \\
\text { lidad }\end{array}$ & 44 & 0.912 & 9.12 & 4 \\
\hline Falta de asistencia a clase & 40 & 0.829 & 8.29 & 5 \\
\hline Técnicas de estudio inadecuadas & 40 & 0.829 & 8.29 & 6 \\
\hline Dificultad de la materia & 39 & 0.809 & 8.09 & 7 \\
\hline Excesiva exigencia del profesorado & 38 & 0.788 & 7.88 & 8 \\
\hline No cursar la carrera en 1 ${ }^{a}$ opción & 37 & 0.767 & 7.67 & 9 \\
\hline $\begin{array}{l}\text { Asignatura impartida por más de } \\
\text { dos profesores }\end{array}$ & 35 & 0.726 & 7.26 & 10 \\
\hline Falta coordinación entre asignaturas & 30 & 0.622 & 6.22 & 11 \\
\hline Escasas perspectivas laborales & 29 & 0.601 & 6.01 & 12 \\
\hline Total & 482 & & & \\
\hline
\end{tabular}

Se consideran neutralizadas, en los expertos del Delphi, las variables sexo y años de experiencia docente. En el Tabla 3 se pueden observar las puntuaciones dadas por los expertos en la ronda final del Delphi.

Tabla 3. Medias y desviaciones típicas de las preguntas cerradas del cuestionario

\begin{tabular}{lllll}
\hline & Válidos & Perdidos & Media & $\begin{array}{c}\text { Desviación } \\
\text { Típica }\end{array}$ \\
\hline $\begin{array}{l}\text { 1. Considera que se han cumplido los } \\
\text { objetivos previstos en el programa de } \\
\text { la asignatura }\end{array}$ & 10 & 0 & 3.20 & .837 \\
\hline $\begin{array}{l}\text { 2. Considera que el estudiante es com- } \\
\text { petente para la aplicación de los prin- } \\
\begin{array}{l}\text { cipios básicos de la teoría del currículo } \\
\text { en cualquier contexto de formación }\end{array}\end{array}$ & 10 & 0 & 3.40 & .548 \\
\hline
\end{tabular}


Análisis de los factores del rendimiento académico de los estudiantes de Pedagogía desde la técnica de grupo nominal y método Delphi

Margarita R. Rodríguez-Gallego

Válidos Perdidos Media

Desviación Típica

3. Considera que el estudiante es competente para la identificación, localización, análisis y gestión de informa$\begin{array}{llll}10 & 0 & 3.60 & 1.140\end{array}$ ción y documentación pedagógica.

4. Considera que el estudiante es competente para diseñar programas $y / o$ planes de intervención educativa (planes de formación, procesos de mejora 10 $0 \quad 3.00$ .000 institucional, actuación pedagógica, actuación social,...)

5. Considera que el estudiante es competente para desarrollar programas $y / o$ planes de intervención educativa (planes de formación, procesos de mejora

$\begin{array}{llll}10 & 0 & 2.80 & .837\end{array}$
institucional, actuación pedagógica, actuación social,...)

6. Considera que el estudiante es competente para organizar y coordinar la utilización y el funcionamiento de los $\begin{array}{llll}10 & 0 & 3.40 & .548\end{array}$ recursos propios de una institución de formación o educación

7. Ha prestado el debido asesoramiento y apoyo a los estudiantes para el $\begin{array}{llll}10 & 0 & 4.40 & .548\end{array}$ estudio de la asignatura

8. Cuál es su valoración sobre el desarrollo de los créditos prácticos de la asignatura

9. Se considera satisfecha/o con las estrategias metodológicas utilizadas

$\begin{array}{lll}10 & 0 & 4.20\end{array}$

10. Se considera satisfecha/o con los resultados finales de la evaluación

$\begin{array}{llll}10 & 0 & 3.40 \quad 1.140\end{array}$

11. Ha tenido buena acogida la asignatura por los estudiantes $\begin{array}{lll}10 & 0 & 3.80\end{array}$ .447

12. Exprese su valoración global del curso 
Análisis de los factores del rendimiento académico de los estudiantes de Pedagogía desde la técnica de grupo nominal y método Delphi

Margarita R. Rodríguez-Gallego

Para facilitar el análisis se dividieron las preguntas en tres bloques: ítems referidos al programa de la asignatura $(1,7,8,9$ y 10$)$, competencias adquiridas por los estudiantes $(2,3,4,5$ y 6), y valoración global de la asignatura (11 y 12$)$.

Al agrupar las preguntas referidas al primer bloque, se puede apreciar que los profesores consideran que no se han cumplido todos los objetivos propuestos en el programa. Están conformes con las estrategias metodológicas desarrolladas en el curso, pero estiman que la evaluación puede mejorarse.

Al analizar las competencias adquiridas por los estudiantes, los docentes consideran que están relativamente capacitados para la aplicación de los principios estudiados en la asignatura, la búsqueda de información y el diseño de programas y/o planes de intervención educativa (planes de formación, procesos de mejora institucional, actuación pedagógica, actuación social,..), pero no tanto para su implementación en instituciones de formación o educación.

En cuanto a la valoración global de la asignatura se sitúa en un rango medio.

Para la pregunta abierta sobre las razones argumentadas para el alto índice de suspensos y no presentados, el estadístico utilizado ha sido un análisis de frecuencias. Consideran que son debidos a la dificultad de la materia, faltan técnicas de estudio, desmotivación y excesivo número de asignaturas, por este orden.

\section{Conclusiones.}

Una vez presentado el análisis de los resultados en el apartado anterior, se resumen las conclusiones más significativas de la investigación y un plan de apoyo para la mejora del rendimiento académico de los estudiantes universitarios.

En primer lugar se comentan los hallazgos más relevantes extraídos de la participación de los estudiantes en la Técnica de Grupo Nominal. Sobre la apreciación de los factores que inciden en su rendimiento, argumentan el siguiente orden:

Factores pedagógico/metodológicos:

- Desproporcionado número de trabajos (1) 
- Asignatura muy teórica (2)

- Dificultad de la materia (7)

- Excesiva exigencia de los profesores (8)

Factores actitudinales:

- Falta de autocontrol y responsabilidad (4)

- Técnicas de estudio inadecuadas (6)

- Escasas perspectivas laborales (12)

Factores institucionales:

- Excesivo número de asignaturas (3)

- Asignatura impartida por más de dos profesores en un mismo grupo (10)

- Falta coordinación entre las asignaturas (11)

Factores académicos:

- Falta de asistencia a clase (5)

- No cursar la carrera en $1^{a}$ opción (9)

Los datos revelan que los estudiantes achacan su bajo rendimiento, en un primer momento a los profesores que imparten la asignatura, seguido de su falta de actitudes para el estudio. Los estudiantes demandan más tiempo para asimilar los conocimientos y realización de créditos prácticos, así como una selección de prácticas más acordes con el programa, explicaciones más pausadas, metodologías más activas e inadecuado sistema de evaluación. Estos resultados coinciden plenamente con los hallazgos encontrados en los estudios de Akiri y Ugborugbo (2009) y Yáñiz (2006).

Los factores institucionales se sitúan en tercer lugar y como una variable relevante el no asistir a clase afecta a un buen rendimiento en la asignatura. Este hecho es constatado igualmente en las investigaciones realizadas por Devadoss y Foltz (1996), Durden y Ellis (1995), Park y Kerr (1990), Karemera (2003), Harb y El-Shaarawi (2007) y Tejedor y Valcárcel (2007). Es Ilamativo el hecho que no consideren el factor cognitivo como determinante del rendimiento. Esto puede ser debido a que no es una carrera muy difícil y se muestran capacitados para finalizarla con éxito.

Los profesores participantes, considerados globalmente, opinan que la variable que más incide en el bajo rendimiento es la dificultad de la materia (variable pedagógica/metodológica), la falta de técnicas de estudio (actitudinal), desmotivación (actitudinal) y excesivo número de asignaturas (institucional). Estos datos son corroborados por la investigación realizada 
Análisis de los factores del rendimiento académico de los estudiantes de Pedagogía desde la técnica de grupo nominal y método Delphi

Margarita R. Rodríguez-Gallego

por Tejedor y Valcárcel (2007) en la Universidad de Salamanca. Implícitamente consideran que ellos son los causantes del bajo rendimiento pues se han cumplido parcialmente los objetivos del programa, los alumnos no son competentes para el desarrollo de programas y/o planes de intervención educativa (planes de formación, procesos de mejora institucional, actuación pedagógica, actuación social,..) y están insatisfechos con los resultados finales del rendimiento académico de los estudiantes.

\subsection{Propuestas de mejora.}

Sería conveniente, respetando los descriptores establecidos, dotar a la asignatura de mayor plasticidad para establecer una mayor vinculación entre teoría y práctica. Los docentes de la asignatura deben insistir no sólo en el diseño sino también la implementación de programas y planes de intervención educativa.

Es importante, en un futuro próximo, crear un plan de apoyo académico para evitar las elevadas tasas de abandono y para estimular el rendimiento en la asignatura. Para ello es preciso cambios en la institución, profesores y estudiantes.

La institución debería revisar los planes de estudio con la posibilidad de incorporar esta asignatura como anual, potenciar la coordinación de los programas de las asignaturas del Grado de Pedagogía, nuevos criterios para elegir horarios en los Departamentos y organizar cursos para aprender técnicas de estudio.

Los alumnos, igualmente, deberían mejorar tanto sus hábitos y técnicas de estudio como sus actitudes de responsabilidad y esfuerzo, además de mayor exigencia al alumnado para llevar a cabo una asistencia regular a las clases, limitando al máximo el absentismo de los estudiantes sin causas justificadas.

Por su parte, los docentes deben ser conscientes de la importancia de su formación pedagógica, haciendo hincapié en la adquisición de estrategias, técnicas de motivación y sistemas de evaluación centrados en el estudiante.

\subsection{Limitaciones del estudio.}

Como limitación del estudio se destaca la falta de participación de los estudiantes seleccionados en el rango de menores de 20 años y la 
Análisis de los factores del rendimiento académico de los estudiantes de Pedagogía desde la técnica de grupo nominal y método Delphi

Margarita R. Rodríguez-Gallego

complejidad del método Delphi para cumplir con rigurosidad los requerimientos metodológicos.

\section{Referencias bibliográficas.}

Abdullah, M.M. e Islam, R. (2011). Nominal Group Technique and its Applications in Managing Quality in Higher Education. Managing Quality in Higher Education, 5(1), 81-99.

Adunola, O. (2011). An Analysis of the Relationship Between Class Size and Academic Performance of Students. Nigeria: Ego Booster Books.

Aharony, N. y Bronstein, J. (2013). A Delphi Investigation into the Future of E-learning. Procedia-Social and Behavioral Sciences 83, 911-914. doi: 10.1016/j.sbspro.2013.06.170

Akiri, A.A. y Ugborugbo, N.M. (2009). Teachers' Effectiveness and Students' Performance in Public Secondary Schools in Delta State, Nigeria. Stud Home Comm Sci, 3(2), 107-113.

Alonso, J. (2005). Motivación para el aprendizaje: la perspectiva de los alumnos, en La orientación escolar en centros educativos (pp. 209-242). Madrid: MEC.

Al-Mutairi, A. (2011). Factors Affecting Business Students' Performance in Arab Open University: Case of Kuwait. International Journal of Business and Management, 6(5), 146-155.

Aripin, R., Mahmood, Z., Rohaizad, R., Yeop, U., y Anuar, M. (2008). Students' learning styles and academic performance. 22nd Annual SAS Malaysia Forum, 15th July 2008, Kuala Lumpur Convention Center, Kuala Lumpur, Malaysia.

Balasubramanian, R. y Agarwal, D. (2012). Delphi Technique- A Review. Journal of Public Health Dentistry, 3(2), 16-25.

Bernal-Delgado, E., Peiró, S. y Sotoca, R. (2006). Prioridades de investigación en servicios sanitarios en el Sistema Nacional de Salud. Una aproximación por consenso de expertos. Gaceta Sanitaria, 20(4), 287-294.

Bharadwaj, B.K. y Pal, S. (2011). Mining Educational Data to Analyse Students' Performance. International Journal of Advanced Computer Science and Applications, 2(6), 63-69.

Cantón, I, Valle, R.E. y Arias, A.R. (2008). Calidad de la docencia universitaria: procesos clave. Educatio Siglo XXI, 26, 121-160.

Devadoss, S. y Foltz, J. (1996). Evaluation of factors influencing students attendance and performance. American Journal of Agricultural Economics, 78(3), 499-507.

Díaz, A. (2003). Personal, family, and academic factors affecting low achievement in secondary school. Electronic Journal of Research in Educational Psychology and Psychopedagogy, 1(1), 43-66.

Durden, G.C. y Ellis, L.V. (1995). The effects of attendance on student learning in principles of economics. American Economic Review, 85(2), 343-346.

Durr, C. (1999). El-S factors affecting student performance in principles of macroeconomics courses. Issues in Political Economy, 8. Consultado en fecha 30-07-2013 en: http://org.elon.edu/ipe/durr.pdf 
Análisis de los factores del rendimiento académico de los estudiantes de Pedagogía desde la técnica de grupo nominal y método Delphi

Margarita R. Rodríguez-Gallego

García, M. y Suárez, M. (2013). Empleo del método Delphi en investigaciones sobre salud publicadas en revistas científicas cubanas. Revista cubana de información en Ciencias de la Salud, 24(2), 133-144.

Harb, N. y El-Shaarawi, A. (2006). Factors affecting student performance. Munich Personal RePEc Archive Paper No. 13621. Consultado en fecha 14-07-2013 en: http:// mpra.ub.uni-muenchen.de/13621/

Ganyaupfu, E.M. (2013). Factors Influencing Academic Achievement in Quantitative Courses among Business Students of Private Higher Education Institutions. Journal of Education and Practice, 4(15), 57-65.

Kang'ahi, M., Indoshi, F.C., Okwach, T.O. y Osido, J. (2012). Teaching Styles and Learners' Achievement in Kiswahili Language in Secondary Schools. International Journal of Academic Research in Progressive Education and Development, 1(3), 62-87.

Karemera, D. (2003). The Effects of Academic Environment and Background Characteristics on Students' Satisfaction and Performance: The Case of South Carolina State University's School of Business. College Student Journal, 37(2), 298-11.

Keeney, S., Hasson, E. y McKenna, H. (2001). A critical review of the Delphi technique as a research methodology for nursing. International Journal of Nursing Studies, 38, 195-200.

Keil, J. y Partell, P. (1997). The effect of class size on student performance and retention at Binghamton University, office of Budget and Institutional Research, Binghamton University. Consultado en fecha 16-07-2013 en: http://buoir.binghamton.edu/papers/ Class_size_jkpp1997.pdf.

Landeta, J. (2006). Current validity of the Delphi method in social sciences. Technological Fore-casting \& Social Change, 73, 467-482.

Lassibille, G. (2011). Student Progress in Higher Education: What We Have Learned from Large-Scale Studies. The Open Education Journal, 4, 1-8.

Mira, J.J., Galán, M., Van Kemenade, E. Marzo, J.C., Blaya, I. y Pérez Jover, M.V. (2012). Retos para el gobierno de las Universidades en el marco del EEEE. Revista de Educación, 357, 445-465. doi: 10-4438/1988-592X-RE-2010-357-068

Montero, E., Villalobos, J. y Valverde, A. (2007). Factores institucionales, pedagógicos, psicosociales y sociodemográficos asociados al rendimiento en la Universidad de Costa Rica: un estudio multinivel. RELIEVE, 13 (2), 215-234. Consultado en fecha 2206-2013 en: www.uv.es/RELIEVE/v13n2/RELIEVEv13n2_5.htm

Morales, A.M. y Fraca, L. (2002). Hacia una política educativa para la enseñanza y el aprendizaje de la lengua escrita en sordos. Investigación y Posgrado, 17(2) Consultado en fecha 26-07-2013 en: http://www.scielo.org.ve/scielo.php?script=sci_ arttext\&pid=S1316-00872002000200004\&lng=en\&nrm=iso\&ignore $=$. html

Omrod, J.E. (2008). Educational psychology: developing learners. Sixth Edition. Upper Saddle River. New Jersey: Pearson Education.

Park, K.H. y Kerr, P.M. (1990). Determinants of academic performance: A multinomial logit approach. Journal of Economic Education, 21(2), 101-111.

Pashler, H., McDaniel, M., Rohrer, D. y Bjork, R. (2008). Learning styles: Concepts and evidence. Psychological Science in the Public Interest, 9(3), 106-119.

Rhodd, R., Schrouder, S. y Allen, M. (2000). Does the performance on Principles of 
Análisis de los factores del rendimiento académico de los estudiantes de Pedagogía desde la técnica de grupo nominal y método Delphi

Margarita R. Rodríguez-Gallego

Economics courses affect the overall academic success of undergraduate business majors?. International Review of Economics Education. Consultado en fecha 12-062013 en: http://www.economicsnetwork.ac.uk/iree/v8n1/rhodd.pdf

Rodríguez-Gallego, M. (2007). Educción de Conocimientos de los Estudiantes Universitarios: la Técnica de Grupo Nominal y el Estudio Delphi. (pp. 389-402). En: Experiencia de Innovación Universitaria (II). Sevilla: Instituto de Ciencias de la Educación de la Universidad de Sevilla.

Romer, D. (1993). Do students go to class? Should they? Journal of Economic Perspectives, 7(3), 167-174.

Sánchez, J., Landeta, J. e Iturralde, T. (2012). La interacción sistema familiar y sistema empresa como fuente de ventaja competitiva. TELOS. Revista de Estudios Interdisciplinarios en Ciencias Sociales, 14(1), 56-82.

Sandrey, M.A. y Bulger, S.M. (2008). The Delphi Method: An Approach for Facilitating Evidence Based Practice in Athletic Training. Athletic Training Education Journal, 3(4), 135-142.

Silva, E.T., Nunes, M.F., Queiroz, M.G. y Leles, C.R. (2010). Factors Influencing Students' Performance in Brazilian Dental School. Brazilian Dental Journal, 21(1), 80-86.

Smith, J. y Naylor, R. (1993). Determinants of degree performance in UK universities: a statistical analysis of the 1993 student cohort. Consultado en fecha 16-07-2013 en: http://www2.warwick.ac.uk/fac/soc/economics/staff/academic/naylor/publications/ obes2001.pdf

Tejedor, F.J. y García-Valcárcel, A. (2007). Causas del bajo rendimiento del estudiante (en opinión de los profesores y alumnos). Propuestas de mejora en el marco del EEES. Revista de Educación, 342, 443-473.

Van der Schaaf, M.F. y Stokking, K.M. (2011). Construct Validation of Content Standards for Teaching. Scandinavian Journal of Educational Research, 55(3), 273-289. doi. 10.1080/00313831.2011.576878

Van der Waal, C.S. y Uys, J.M. (2009). Applying the nominal group technique in an employment relations confl ict situation: A case study of a university maintenance section in South Africa. SA Journal of Human Resource Management/SA Tydskrif vir Menslikehulpbronbestuur,7(1), 137-143. doi:10.4102/sajhrm.v7i1.232

Yániz, C. (2006). Planificar la enseñanza universitaria para el desarrollo de competencias. Educatio Siglo XXI, 24, 17-34.

Yáñez, R. y Cuadra, R. (2008). La técnica Delphi y la investigación en los servicios de salud. Ciencia y Enfermería, XIV(1), 9-15.

Yousuf, M.I. y Ranjha, A.N. (2011). A Study of Non-cognitive Variables of Academic Achievement at Higher Education: Nominal Group Study. Asian Social Science, 7(7), 53-58. doi:10.5539/ass.v7n7p53 
